\title{
On Lie Derivations of 3-Graded Algebras
}

\author{
A. J. CALDERÓN MARTÍN AND C. MARTÍN GONZÁLEZ
}

\begin{abstract}
We prove that any graded Lie derivation on certain 3-graded associative algebras is a graded derivation. As an application we show that the graded Lie derivations on infinite dimensional topologically simple 3 -graded associative $H^{*}$-algebras are also graded derivations.
\end{abstract}

\section{INTRODUCTION}

Over the years, there has been considerable effort made and success in studying the structure of derivations and Lie derivations of rings $([2,3])$, and Banach algebras $([12,15,16])$. The 3 -graded algebras have been considered in the literature with emphasis on their connections with Jordan pairs and the associated groups $([13,14,17])$, we have also introduced in [5] techniques of derivations and 3-graded algebras in the treatment of problems of Lie isomorphisms. We are interested in investigating the Lie derivations on 3-graded associative algebras. We recall that given a unitary commutative ring $K$, a 3-graded $K$-algebra $A$ is a $K$-algebra which splits into the direct sum $A=A_{-1} \oplus A_{0} \oplus A_{1}$ of nonzero $K$-submodules satisfying $A_{0} A_{i}+A_{i} A_{0} \subset A_{i}$ for all $i \in\{-1,0,1\}, A_{-1} A_{1}+A_{1} A_{-1} \subset A_{0}$ and $A_{1} A_{1}=A_{-1} A_{-1}=0$. A linear mapping $D$ on a 3 -graded associative algebra $A$ such that $D\left(A_{i}\right) \subset A_{i}, i \in\{-1,0,1\}$, is called a graded derivation if satisfies $D(x y)=D(x) y+x D(y)$ for all $x, y \in A$, and it is called a graded Lie derivation if $D([x, y])=[D(x), y]+[x, D(y)]$ holds for all $x, y \in A$. Here and subsequently, the bracket denotes the Lie product, $[x, y]=x y-y x$ on $A$. From now on $A$ will denote

2000 Mathematics Subject Classification. Primary 17B40, 17B70; Secondary $46 \mathrm{~K} 70$.

The authors are supported in part by the PCI of the spanish Junta de Andalucía 'Estudio analítico-algebraico de sistemas triples y de pares en diferentes clases de estructuras no asociativas', by the PAI of the spanish Junta de Andalucía with project number FQM-0125 and by the the Spanish DGICYT. 
a 3-graded associative algebra defined over a field $K$ of characteristic not 2. We define the annihilator of $A$ as the ideal given by $\operatorname{Ann}(A)=\{x \in A: x y=y x=0$ for all $y \in A\}$. Our purpose is to show the following theorem

Theorem 1. Let $D$ be a graded Lie derivation on a semiprime 3-graded associative algebra $A=A_{-1} \oplus A_{0} \oplus A_{1}$ such that $A_{0}=$ $\left[A_{-1}, A_{1}\right]$. Then $D$ is a 3-graded derivation.

Corollary 1. Let $D$ be a graded Lie derivation on an infinite dimensional topologically simple 3-graded associative $H^{*}$-algebra $A$. Then $D$ is a 3-graded derivation.

\section{The Theorem}

Given a 3-graded associative algebra, we have clearly that $\left(A_{-1}, A_{1}\right)$ is an associative pair with respect to the triple products $\langle x, y, z\rangle^{\sigma}=$ $x y z$ for $\sigma= \pm$, with $x, z \in A_{-1}$ and $y \in A_{1}$ if $\sigma=+$, and with $x, z \in A_{1}$ and $y \in A_{-1}$ if $\sigma=-$. If $P=\left(P^{+}, P^{-}\right)$is an associative pair isomorphic to the associative pair $\left(A_{-1}, A_{1}\right)$, we shall say that $A$ is a 3 -graded algebra envelope of $P$ if $A_{0}=A_{-1} A_{1}+A_{1} A_{-1}$. An envelope $A$ is tight if

$$
\left\{x_{0} \in A_{0}: x_{0} A_{\sigma}=A_{\sigma} x_{0}=0, \sigma= \pm 1\right\}=0 .
$$

A derivation $D$ on a, non-necessarily associative, pair $V=\left(V^{+}, V^{-}\right)$ is a couple of linear mappings $D=\left(D^{+}, D^{-}\right), D^{\sigma}: V^{\sigma} \rightarrow V^{\sigma}$, satisfying

$$
\begin{aligned}
& D^{\sigma}\left(<x^{\sigma}, y^{-\sigma}, z^{\sigma}>\right)=<D^{\sigma}\left(x^{\sigma}\right), y^{-\sigma}, z^{\sigma}> \\
& +<x^{\sigma}, D^{-\sigma}\left(y^{-\sigma}\right), z^{\sigma}>+<x^{\sigma}, y^{-\sigma}, D^{\sigma}\left(z^{\sigma}\right)>
\end{aligned}
$$

for any $x^{\sigma}, z^{\sigma} \in V^{\sigma}, y^{-\sigma} \in V^{-\sigma}$ and $\sigma= \pm$.

Proposition 1. Let $P=\left(P^{+}, P^{-}\right)$be an associative pair, let $D$ be a derivation of $P$, and let $A$ be a 3-graded tight algebra envelope of $P$. Then there is a unique graded derivation $D^{\prime}$ on $A$ extending $D$.

Proof. As $A=P^{+} \oplus\left(P^{+} P^{-}+P^{-} P^{+}\right) \oplus P^{-}$, we can define first

$$
D^{\prime}: P^{+} \oplus\left(P^{+} P^{-}+P^{-} P^{+}\right) \oplus P^{-} \rightarrow A
$$

by writing $D^{\prime}(x):=D^{\sigma}(x)$ for all $x \in P^{\sigma}, \sigma= \pm$, and 


$$
\begin{array}{r}
D^{\prime}\left(\sum_{j}\left(x_{j} y_{j}+u_{j} v_{j}\right)\right):=\sum_{j}\left(D^{+}\left(x_{j}\right) y_{j}+x_{j} D^{-}\left(y_{j}\right)\right. \\
\left.+D^{-}\left(u_{j}\right) v_{j}+u_{j} D^{+}\left(v_{j}\right)\right)
\end{array}
$$

for arbitrary $x_{j}, v_{j} \in P^{+}$and $y_{j}, u_{j} \in P^{-}$. The definition is correct since if $\sum_{j}\left(x_{j} y_{j}+u_{j} v_{j}\right)=0$. Letting

$$
z:=\sum_{j}\left(D^{+}\left(x_{j}\right) y_{j}+x_{j} D^{-}\left(y_{j}\right)+D^{-}\left(u_{j}\right) v_{j}+u_{j} D^{+}\left(v_{j}\right)\right),
$$

the equations

$$
D^{+}\left(\sum_{j}\left(x_{j} y_{j}+u_{j} v_{j}\right) x\right)=0 \quad \text { and } \quad D^{-}\left(\sum_{j}\left(x_{j} y_{j}+u_{j} v_{j}\right) y\right)=0
$$

for any $x \in P^{+}$and any $y \in P^{-}$imply $z P^{\sigma}=0, \sigma= \pm$. In a similar way we have $P^{\sigma} z=0$. Hence $z=0$. The fact that $D^{\prime}$ is a derivation is easy to check and the proof is complete.

Proof of Theorem 1. As $A$ is a 3 -graded associative algebra, we find that the pair

$$
J:=\left(\left(A_{-1}, A_{1}\right),\{\cdot, \cdot, \cdot\}^{\sigma}\right)
$$

is a Jordan pair in the sense of [10] with respect to the triple products $\{x, y, z\}^{\sigma}=[[x, y], z]$ for $\sigma= \pm$, with $x, z \in A_{-1}, y \in A_{1}$ if $\sigma=+$ and $x, z \in A_{1}, y \in A_{-1}$ if $\sigma=-$. Hence $(D, D)$ is a derivation of $J$. It is proved in [11] that any Jordan derivation on a semiprime associative pair over a field $K$ of characteristic not 2 is an associative derivation. As the 3-graduation of $A$ also implies $\{x, y, z\}^{\sigma}=x y z+z y x$, the above result gives us that $(D, D)$ is a derivation of the associative pair $\left.P:=\left(\left(A_{-1}, A_{1}\right),<\cdot, \cdot, \cdot\right\rangle^{\sigma}\right)$ being $\langle\cdot, \cdot, \cdot\rangle^{\sigma}=x y z$. It is easy to check that $A$ is a 3-graded tight algebra envelope of $P$, hence Proposition 1 shows that $(D, D)$ extends uniquely to a derivation $D^{\prime}$ on $A$. We assert that $D=D^{\prime}$. Indeed, $D(x)=D^{\prime}(x)$ for any $x \in A_{-1} \cup A_{1}$ and chosen any $x_{0} \in A_{0}$, the condition $A_{0}=\left[A_{-1}, A_{1}\right]$ gives us $D\left(x_{0}\right)=D^{\prime}\left(x_{0}\right)$. The proof is complete.

In order to prove Corollary 1 , we recall that an $H^{*}$-algebra $A$ over $K, K=\mathbb{R}$ or $K=\mathbb{C}$, is a non-necessarily associative $K$-algebra whose underlying vector space is a Hilbert space with inner product 
$(\cdot \mid \cdot)$, endowed either with a linear map if $K=\mathbb{R}$ or with a conjugatelinear map if $K=\mathbb{C}, *: A \rightarrow A\left(x \mapsto x^{*}\right)$, such that $\left(x^{*}\right)^{*}=x$, $(x y)^{*}=y^{*} x^{*}$ for any $x, y \in A$ and the following hold

$$
(x y \mid z)=\left(x \mid z y^{*}\right)=\left(y \mid x^{*} z\right)
$$

for all $x, y, z \in A$. The map $*$ will be called the involution of the $H^{*}$-algebra. The continuity of the product of $A$ is proved in [8]. We call the $H^{*}$-algebra $A$, topologically simple if $A^{2} \neq 0$ and $A$ has no nontrivial closed ideals. $H^{*}$-algebras were introduced and studied by Ambrose [1] in the associative case, and have been also considered in the case of the most familiar classes of nonassociative algebras $[4,6,8,9]$ and even in the general nonassociative context $[7,15]$. In [8] it is proved that any $H^{*}$-algebra $A$ with continuous involution splits into the orthogonal direct sum $A=\operatorname{Ann}(A) \perp \overline{\mathcal{L}\left(A^{2}\right)}$, where $\operatorname{Ann}(A)$ denotes the annihilator of $A$ defined as in $\S 1$, and $\overline{\mathcal{L}\left(A^{2}\right)}$ is the closure of the vector span of $A^{2}$, which turns out to be an $H^{*}$ algebra with zero annihilator. Moreover, each $H^{*}$-algebra $A$ with zero annihilator satisfies $A=\overline{\perp I_{\alpha}}$ where $\left\{I_{\alpha}\right\}_{\alpha}$ denotes the family of minimal closed ideals of $A$, each of them being a topologically simple $H^{*}$-algebra. We also recall that any derivation on arbitrary $H^{*}$-algebras with zero annihilator is continuous [15].

Proof of Corollary 1. The structure theories of topologically simple associative and Lie $H^{*}$-algebras given in [8] and [6] respectively imply that the antisymmetrized Lie $H^{*}$-algebra $A^{-}$of $A$ is also a topologically simple Lie $H^{*}$-algebra. Hence $A_{0}=\overline{\left[A_{-1}, A_{1}\right]}$. There is not any problem in arguing as in Theorem 1 to prove that $D=D^{\prime}$ on $A_{1} \oplus\left[A_{-1}, A_{1}\right] \oplus A_{1}$. By [15], $D$ and $D^{\prime}$ are continuous and therefore $D=D^{\prime}$ on $A=A_{1} \oplus \overline{\left[A_{-1}, A_{1}\right]} \oplus A_{1}$.

\section{REFERENCES}

[1] W. Ambrose, Structure theorems for a special class of Banach algebras, Trans. Amer. Math. Soc. 57 (1945), 364-386.

[2] R. Banning and M. Mathieu, Commutativity-preserving mappings on semiprime rings, Comm. Algebra 25 (1997), 247-265.

[3] M. Brešar, Commuting traces of biadditive mappings, commutativitypreserving mappings and Lie mappings, Trans. Amer. Math. Soc. 335 (1993) 525-546.

[4] M. Cabrera, J. Martínez and A. Rodríguez, Structurable $H^{*}$-algebras, J. Algebra 147, no. 1 (1992), 19-62. 
[5] A. J. Calderón and C. Martín, Lie isomorphisms on $H^{*}$-algebras, Comm. Algebra. In press.

[6] J. A. Cuenca, A. García and C. Martín, Structure theory for $L^{*}$-algebras, Math. Proc. Cambridge Philos. Soc. 107, no. 2 (1990), 361-365.

[7] J. A. Cuenca and A. Rodríguez, Isomorphisms of $H^{*}$-algebras, Math. Proc. Cambridge Philos. Soc. 97, (1985), 93-99.

[8] J. A. Cuenca and A. Rodríguez, Structure theory for noncommutative Jordan $H^{*}$-algebras, 106 (1987), 1-14.

[9] Devapakkian, C. Viola and P. S. Rema, Hilbert space methods in the theory of Jordan algebras, Math. Proc. Cambridge Philos. Soc. 78 (1975), 293-300.

[10] O. Loos, Jordan pairs, Lecture Notes in Mathematics, Springer-Verlag, Berlin-New York, vol. 460, (1976), 307-319.

[11] M. Marhinine, C. Zarhouti, Jordan derivations on associative pairs. Preprint, University of Tetouan.

[12] M. Mathieu, "Where to find the image of a derivation", Banach Center Publications, Vol. 30, pp. 237-249, PWN, Warsaw, 1994.

[13] K. Meyberg, "Lectures on algebraic and triple systems", Lecture notes, University of Virginia, Chalottesville, 1976.

[14] E. Neher, Generators and relations for 3-graded Lie algebras, J. Algebra 155 (1993), 1-35.

[15] A. R. Villena, Continuity of derivations on $H^{*}$-algebras, Proc. Amer. Math. Soc. 122 (1994), 821-826.

[16] A. R. Villena, Lie derivations on Banach algebras, J. Algebra 226 (2000), 390-409.

[17] E. Zelmanov, Lie algebras with a finite grading, Math. USSR-SB 52 (1985), 347-385.

\begin{abstract}
A. J. Calderón Martín,
Departamento de Matemáticas,

Universidad de Cádiz,

11510 Puerto Real, Cádiz, Spain

ajesus.calderon@uca.es
\end{abstract}

\author{
C. Martín González, \\ Departamento de Algebra, \\ Geometría y Topología, \\ Universidad de Málaga, \\ Apartado 59, \\ 29080 Málaga, Spain.
}

Received on 20 October 2001 and in revised form on 13 July 2002. 